\title{
Dagli archivi dell'accademia spagnola. Freud e la messa in intreccio
}

Mario Lavagetto

«Simply the thing I am

Shall make me live.»

Shakespeare

La prima tra le grandi amicizie che costellano la vita di Freud, e ne segnano il percorso, è quella con Eduard Silberstein. Le lettere che ci restano coprono un arco di dieci anni e costituiscono una preziosa testimonianza non solo sulla vita, ma anche sulla formazione culturale e intellettuale di Freud. Nel loro insieme sono (possono essere considerate) i verbali di un'associazione segreta, stipulata tra due adolescenti e retta da statuti minuziosi e circostanziati per proteggerla dall'esterno. Una società che i due fondatori hanno battezzato Accademia Spagnola, assumendo a loro volta, secondo le regole, nomi fittizi e derivati da una delle Novelle esemplari di Cervantes, Matrimonio degli inganni e colloquio dei cani: Silberstein è Berganza e Freud è Cipio.

La distribuzione delle parti può risultare suggestiva: nel racconto di Cervantes, l'alfiere Campuzano riferisce all'avvocato Peralta il «colloquio» che ha potuto ascoltare durante il suo ricovero nell'Ospedale della Resurrezione a Valladolid e della cui «autenticità» ha dovuto lui stesso capacitarsi: «dato che non può trattarsi di una mia invenzione, sono stato costretto a convincermi, sia pure contro ogni apparenza e a malincuore, che non ho sognato e che i due cani parlavano.» Distesi su vecchie stuoie, si sono raccontati nel cuore della notte la loro esistenza: per primo ha parlato Berganza e l'alfiere ha trascritto fedelmente le sue parole, riservandosi di redigere anche il resoconto della seconda notte (quando Cipio ha assunto il ruolo di narratore), una volta che avrà raggiunto la certezza di essere creduto o, almeno, non disprezzato. Quella certezza, dobbiamo supporre, l'alfiere non riuscì a raggiungerla e non resta ai lettori che il resoconto della prima notte: così la parte di Cipio è quella del cane che ascolta, commenta, chiede chiarimenti, suggerisce, indirizza il discorso del suo interlocutore e lo invita finalmente a «raccontare quel che gli pare e come gli pare», con una licenza non molto diversa da quella che Freud concederà ai propri pazienti.

Nulla ci impedisce di raccogliere il filo della finzione là dove Cervantes lo aveva lasciato cadere. «La prossima notte - aveva detto Cipio congedandosi- se questo gran dono della favella non ci avrà abbandonato, sarà tutta 
mia, e ti racconterò la mia vita.» Le lettere di Freud a Silberstein, così come ci sono pervenute (senza le risposte del suo corrispondente), possono essere considerate, nel giocoso regime dell' Accademia Spagnola, il racconto di quella seconda notte, il racconto che l'erede di Cipio fa della sua esistenza. Che queste lettere, d'altronde, costituiscano agli occhi del giovane Freud una sorta di autobiografia, non è una congettura. Ce lo dice lui stesso, diciottenne, il 13 agosto 1864:

Dio non ha creato il mondo in un secondo per indicare agli uomini che in ogni opera bisogna mostrare un ordine e una successione [...] è lo stesso ordine che devono mostrare le nostre lettere, non un ordine artificiale e privo di vita, ma quello di un'opera d'arte in cui le singole parti non sono soltanto separate le une dalle altre, ma mostrano uno stretto legame interno.

E qualche mese più tardi, dopo avere proposto a Silberstein di trasformare la loro corrispondenza in uno scrupoloso diario giornaliero, Freud ribadirà: "una lettera è sempre una messa in opera delle cosiddette arti liberali.»

Le preoccupazioni (o le tentazioni) letterarie di Freud non si limitano al solo epistolario: in quegli anni scrive racconti, poesie, trattati, e li accumula negli "Archivi» dell' Accademia spagnola, probabilmente sottratti alla nostra curiosità dal rogo a cui Freud racconterà a Martha di avere destinato tutte le sue carte «a disperazione dei futuri biografi.» $\mathrm{Ci}$ resta qualche frammento; un dialogo tra due astri, che al curatore tedesco delle lettere, Walter Boehlich, ha ricordato — non a torto - il clima e il tono delle Operette morali, allora non tradotte in tedesco; un Poema nuziale, scritto con intenzioni scopertamente parodiche in "esametri» inframmezzati da versi di 5 o di 7 piedi, perché non c'è ragione di limitarsi a sei, "se un piede in più $\mathrm{o}$ in meno, per diminuzione $\mathrm{o}$ sottrazione, può renderli più scorrevoli.» Restano, soprattutto, tracce di una volontà di scrittura molto determinata e sostenuta da un talento tanto forte da sorprendere anche chi abbia riconosciuto da tempo le qualità stilistiche di Freud.

Affiora, in una circostanza, una formula perentoria e citata tra virgolette: il problema, si dice, "è quello di dare artisticamente forma al vissuto.» [das Erlebt künstlerisch zu gestalten]. La fonte di Freud, se esiste, non è stata identificata e tuttavia non è difficile riconoscere in queste parole un prodotto di quella sorta di «aristotelismo naturalistico», che sembra affiorare talvolta nella narrativa di fine secolo: die Fabeln (die Sujets) gestalten è traduzione corrente

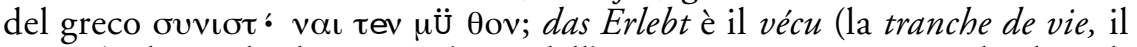
vissuto), che negli ultimi trent'anni dell'Ottocento assume quasi il valore di una parola d'ordine. Non ci sarebbe alcuna scorrettezza a riutilizzare quella formula per definire il lavoro compiuto da Freud al momento di redigere e di dare forma ai suoi casi clinici, anche se — con ogni probabilità- lo stesso Freud avrebbe respinto — come vedremo- l'avverbio künstlerisch, che gli sarebbe apparso insieme pericoloso (nelle mani di possibili avversari) e fuorviante sul piano teorico. 
Simili precauzioni erano del tutto superflue nell'ambito dell'Accademia Spagnola e Freud, studente di medicina, non esita a progettare il proprio passaggio alla facoltà di lettere: e questo avviene quando più forte è su di lui l'influenza di Franz Brentano («un uomo, uno scienziato e un filosofo meraviglioso»), di cui segue le lezioni nel semestre invernale del 1874. «E maturata in me la decisione - scrive il 7 marzo 1875 - di sostenere il dottorato in filosofia sulla base di studi filosofici e zoologici.» E un mese dopo, l'11 aprile: «Siamo alle soglie del secondo semestre: una nuova vita per me, in cui sarò prima di tutto filosofo e zoologo, perché seguirò corsi di filosofia, di logica e due di zoologia.» $\mathrm{Si}$ avverte in queste parole l'eco molto netta dell' insegnamento di Brentano che, nell'introduzione al suo Die Psychologie des Aristoteles, pubblicato nel 1866, aveva sostenuto l'esistenza di un rapporto strettissimo tra la logica e la psicologia, rapporto analogo a quello che sussiste «tra la medicina terapeutica e quella parte della scienza naturale che l'epoca moderna ha chiamato con il nome di biologia o di fisiologia (in senso lato).»

Chi conosce l'itinerario biografico di Freud, e la sua opera, è abituato ai rivolgimenti improvvisi. Una parte dell'estate del 1875 la trascorre in Inghilterra a Manchester, in casa del maggiore dei suoi fratelli, Emmanuel. Al suo ritorno scrive una lunghissima lettera a Silberstein datata 9 settembre:

...ti dirò francamente che preferirei vivere là che a Vienna, a dispetto della nebbia e della pioggia, degli abusi alcoolici e del conservatorismo [...] Ti confesserò una cosa: oggi ho più di un ideale; ho aggiunto un ideale pratico al mio ideale teorico degli anni precedenti. L'anno scorso alla domanda: qual è il tuo desiderio più grande? avrei risposto: avere un laboratorio e del tempo libero o una nave sull'oceano, provvista di tutti gli strumenti di cui un ricercatore ha bisogno; ora sono incerto e mi chiedo se non dovrei dire piuttosto: un grande ospedale e abbastanza denaro per arginare o bandire da questo mondo qualcuno dei mali che colpiscono il nostro corpo. [...] Un uomo che godesse di alta considerazione e del sostegno della stampa e dei ricchi potrebbe fare miracoli per attenuare le sofferenze fisiche, se ha sufficiente spirito di ricercatore per impegnarsi in nuove strade terapeutiche.

E più avanti, a sancire la completa rivoluzione delle sue prospettive:

la conoscenza che ho acquisito di opere scientifiche inglesi, avrà come conseguenza di tenermi sempre, nei miei studi, dalla parte degli Inglesi che, ai miei occhi, godono di un pregiudizio altamente favorevole: Tyndall, Huxley, Lyell, Darwin, Thomson, Lockyer. Sono più diffidente che mai nei confronti della filosofia...

Venti giorni dopo, il 1 ottobre, Freud aggiunge un poscritto alla lettera con cui ha inviato a Silberstein il suo Poema nuziale:

Qui si chiude un periodo di formazione, qui seppellisco la bacchetta magica che ha contribuito alla sua creazione, che qui inizi una nuova era, non immischiata con le forze oscure, un'era che non avrà bisogno né di poesia, né di fantasia.» 
Si tratta insieme di un esorcismo e di un proponimento: queste parole — «con un misto di dileggio e di tristezza»— ufficializzano la nascita dello «scienziato positivo», del terapeuta capace di intraprendere nuove strade. Che poi - per annunciare questa nascita - Freud abbia bisogno di ricorrere a una citazione letteraria e di evocare le parole di Prospero all'inizio del quinto atto della Tempesta ("I'll break my staff...»), è sintomatico: è il segno di un tramonto provvisorio, reiteratamente celebrato e mai definitivo.

Tra i documenti dell'Accademia spagnola, di cui Freud comunica a Silberstein lo scherzoso inventario il 21 febbraio 1875, si trova anche una "famosa relazione de mediis quibus in amoribus efficiendis utuntur poetae, che basterebbe da sola a fare di me il felice continuatore di Aristotele.» Purtroppo si è perduta ogni traccia di quello scritto, ma quando Freud -il 16 settembre 1883, in una lunga lettera a Martha- dissotterra, in modo forse preterintenzionale, non certo inconsapevole, la sua bacchetta per raccontare la morte di Nathan Weiss, il discepolo, se non il continuatore di Aristotele, torna ad affacciarsi: e la narrazione si iscrive tra due formule in cui è percepibile un' eco della Poetica.

Giovane medico, assistente alla Clinica neurologica, Weiss si è impiccato alle due del pomeriggio del giorno 13 in un bagno della Landstrasse. «Era sposato da appena un mese e tornato da dieci giorni dal viaggio di nozze.» Ha lasciato due lettere, ma il suo gesto resta, per tutti, inspiegabile: nessuno riesce a immaginare che quell'uomo tanto irrequieto, e con un così profondo gusto per la vita, sia "morto e muto». "Anche ora, dopo aver sentito rotolare le zolle di terra sulla sua bara, non riesco ad abituarmi a quella idea.»

Pareva che, sul piano professionale, Weiss avesse conseguito, o fosse sul punto di conseguire, tutto quanto poteva desiderare: era docente; dirigeva un reparto dell'ospedale; aveva una vasta clientela. Per capire - dice Freud - cosa lo ha spinto a darsi la morte, bisogna cercare nel suo matrimonio.

Non so cosa ti ho raccontato sulla preistoria di questo matrimonio; credo di doverti ripetere tutto quel che so di lui, perché non è morto per caso, piuttosto il suo essere si è compiuto, le sue buone e cattive qualità si sono unite per condurlo alla rovina, la sua vita era come composta da un poeta, e la morte ne fu come la necessaria catastrofe.

Fatta questa premessa, Freud comincia a riferire a Martha «tutto quello che sa» di Weiss: ne nasce uno splendido racconto in cui il narratore, nelle sue vesti di testimone oculare, enuncia i fatti e formula congetture, cercando accanitamente le impronte della necessità. A un rapido ritratto dei genitori, segue un primo profilo di Nathan: contraddizioni, smisurato amore di sé, spregiudicatezza, instancabile e vertiginosa attività per nascondere un vuoto divorante.

$\grave{E}$ un uomo di superficie, in continua fuga dalla realtà, a cui peraltro cerca di abbarbicarsi grazie a un' affannosa ed esasperata attività mimetica: la sua è una forma, per quanto distorta e mascherata, di onnivoro narcisismo. Un Narciso paradossale, tuttavia, teso a evitare la propria immagine come quello 
genialmente reinventato da La Fontaine e che, nel timore di riconoscere la sua inadeguatezza al modello, la sua miseria, la sua cronica indigenza, si ritira dal mondo, determinato a evitare o a infrangere tutti gli specchi per non compromettere l'autoritratto che si è pazientemente costruito e che un soffio d'aria potrebbe dissolvere:

Sembrava che vivesse sempre a porte aperte; solo dopo la sua morte abbiamo capito che nascondeva molte cose.

Freud recupera e mette in ordine i singoli dettagli evitando scrupolosamente, con il suo talento di ritrattista, di fornirne una lettura univoca: il modulo dell'avversativa, esplicito o implicito, è l'indice di un continuo rovesciamento delle apparenze che il narratore propone per non cadere nel tranello delle «porte aperte»; le parole — soprattutto le parole del protagonista, ma non solo le suevengono spesso registrate in presa diretta.

Attorno a Weiss spuntano, così, altri personaggi: il narratore prima di tutto, discreto e onnipresente; poi Breuer, nelle vesti del saggio consigliere; i familiari ; i colleghi; la moglie. Tutto l'organico, insomma, di una compiuta tragedia borghese. La chiave del destino, come Freud ha anticipato fin dalle prime righe, va cercata nel matrimonio che fornisce la stretta finale al racconto:

forse influenzato dalla felicità in amore di quanti gli stavano intorno, cercò anch'egli di procurarsela, cercò e cercò, e non lasciò a se stesso né il tempo né l'opportunità di incontrarla.

Basterebbe la triplice ripetizione del verbo a dimostrare tanto la sofisticata strumentazione retorico-narrativa utilizzata da Freud, quanto le intenzioni della sua scrittura. Intenzioni anche più precise e riconoscibili a partire dal momento in cui il personaggio-Weiss viene posto nella situazione che Freud studierà molti anni più tardi nel saggio Il motivo della scelta degli scrigni : sceglie, Nathan, lo scrigno che contiene la ricchezza, ma anche la morte.

La moglie è una specie di Brünnhilde, fredda ed equilibrata, poco femminile, forse con predisposizioni isteriche, assillata dalla famiglia, ma niente affatto desiderosa di sposarsi. Weiss, che già in altra occasione era stato respinto, non può tollerare l'idea di un rifiuto:

le fece una corte sempre più pressante, regali per migliaia di fiorini, spese una forte somma per il suo corredo, liberò tutti i suoi risparmi per sistemare splendidamente l'abitazione e renderle difficile un diniego.

Il consenso alla fine viene strappato, ma in una forma inquietante: «a questo punto devo sposarmi - dice la donna-, immediatamente o mai più». Weiss non ha esitazioni e il matrimonio viene fissato e celebrato in gran fretta.

«Dopo il viaggio di nozze lo vidi una sola volta, non era solo e non si sbottonò.» Eppure, in quella circostanza, Weiss ha presumibilmente pronunciato 
una frase misteriosa e in apparenza insignificante, che Freud - prima ancora di mettersi a parlare del matrimonio - ha citato tra virgolette, come se in quelle parole si nascondesse un inconfutabile segno di identità:

Non sono uno di quelli che possano passare delle ore a contemplare la superficie di un lago e che riescano a entusiasmarsene.

Cosa significavano quelle parole? Cosa significavano per Weiss? Ma cosa significavano anche per Freud, che se ne serve per provare la superficialità dell'amico, la sua indifferenza, nei "momenti felici», alle bellezze del mondo. Dicevano solo quello? O, in quel punto preciso della «storia», dieci giorni prima del suicidio, non hanno il valore di un segnale?

Può nascere il dubbio che qui il narratore abbia fornito in modo deliberatamente enigmatico un indizio, un reperto inquietante, collocandolo "fuori posto» e lasciando a noi la responsabilità di recuperarlo e di interrogarlo. Ma può anche darsi che in questa circostanza l'istinto del "padre di tutti i racconti» abbia funzionato alle spalle di Freud e lo abbia indotto a salvare un frammento il cui valore, almeno in parte, gli sfuggiva. Una simile, congiunturale incertezza appartiene, tuttavia, al patrimonio dei lettori di racconti e conferma, se ce ne fosse bisogno, la "natura" narrativa e clandestina del testo che stiamo leggendo.

Weiss si impicca il giorno tredici. Qualcuno accusa la moglie. «Io — replica Freud- non ci credo», e chiude il primo anello della sua narrazione:

È morto per un insieme di qualità, per il suo morboso amor proprio, come anche per le sue aspirazioni verso qualcosa di migliore. Sbagliano dunque quelli che hanno cercato le cause della morte al di fuori della storia e della necessità iscritta nella vita di Nathan Weiss.

Hanno sbagliato anche, e soprattutto, le famiglie che si sono scambiate accuse «sul suo cadavere». Il racconto torna ora sui propri passi e così veniamo ricondotti bruscamente al funerale da cui eravamo partiti, dove si chiuderà il secondo anello narrativo:

«...quando la tomba era ancora aperta, si è udito uno stridulo grido di vendetta, così iniquo e brutale che pareva venisse da lui.» Ha preso la parola il dottor Friedmann un parente e un collega del padre: «Se si trova un cadavere e non si sa per mano di chi ha perduto la vita, allora bisogna cercare tra i prossimi, essi sono gli assassini. Ma noi, i suoi genitori e fratelli, non abbiamo versato il suo sangue...", e a questo punto cominciò a chiare lettere ad accusare l'altra famiglia di avere inferto a Nathan il colpo mortale. Parlava con la voce esaltata del fanatico, con la violenza dell'ebreo selvaggio e spietato. Noi tutti eravamo impietriti dall'indignazione e dalla vergogna per i cristiani che erano tra noi.

Ormai a Freud (che aveva raccomandato a Silberstein di costruire le lettere con lo stesso rigoroso ordine con cui era costruito l'universo) non resta che 
il terzo anello da chiudere, l'anello teorico che si intrecciava col primo:

Così la sua morte come la sua vita appaiono modellate con lo stesso impasto, sembra davvero che egli invochi il poeta che lo conservi nella memoria degli uomini.

Finora abbiamo proceduto lungo una linea aneddotica. Ora dobbiamo compiere un passo indietro: prendere «l'anello teorico» e utilizzarlo con qualche libertà per metterne in luce il senso e la portata. Freud ci dice che Weiss non è morto per caso e che per questo la sua vita, composta come da un poeta, è modellata con lo stesso impasto con cui è stata modellata la sua morte. In altri termini è la non casualità a legittimare il segno di uguaglianza tra la vita di Weiss e un'opera d'arte; ed è ancora la non casualità a rendere spiegabile la sua morte che si configura come la «necessaria catastrofe» di quella vita.

Basterebbe che un poeta (con i suoi «doni misteriosi», sulla cui natura Freud eviterà sempre di pronunciarsi) raccogliesse i materiali esposti nella lettera a Martha e, grazie al suo soffio creatore, Weiss troverebbe il proprio posto nella memoria degli uomini. Si potrebbe osservare che la lettera costituisce di per se stessa una garanzia di sopravvivenza e che Freud, scrivendola, dimostra di possedere non solo i doni, ma anche la volontà del Charakterdichter : quello che importa però è che, giunto alla fine, Freud si schernisca. E' come se dicesse: «La vita di Weiss, che si è svolta all'insegna della necessità, era come un'opera d'arte; ma la ricostruzione che io ne ho fornito — questa lettera- non è un'opera d'arte. Il poeta deve ancora venire."

Possiamo sorvolare, almeno per il momento, sulle ragioni della reticenza di Freud, che lo induce a negare ciò che ogni lettore, anche il più malintenzionato, sarebbe pronto a riconoscere, e cioè che la bacchetta magica di Prospero è stata in questa circostanza felicemente, meravigliosamente dissotterrata. Quel che importa, ora, è la poetica implicita di Freud, che — nella sua essenzialitàpotrebbe essere stata ricavata da Aristotele. Il quale non si stanca di sottolineare che una tragedia è perfetta quando le azioni si susseguono le une alle altre

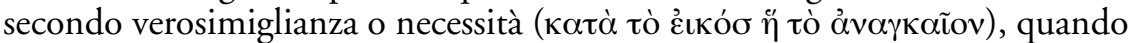
la vicenda non procede in modo episodico e discontinuo e i fatti si convengono reciprocamente, disponendosi in un intreccio ordinato, in modo che i singoli casi «nascano dalla struttura stessa del racconto», poiché è «molto diverso se una cosa accade a causa di un'altra o semplicemente dopo un'altra».

Partendo da questi frammenti di poetica, enunciati nel 1883, si possono formulare alcuni quesiti per una teoria che vedrà la luce solo una quindicina d'anni più tardi, una teoria che:

a) esclude rigorosamente il caso;

b) proprio perché esclude il caso, pensa di potere costruire a ritroso una catena che consentirà di andare dai sintomi alle loro radici etiologiche; di risalire dal dato alle condizioni sotto cui soltanto quel dato è possibile. Si affida, insomma, a un metodo che Kant suggeriva di definire «analitico, meglio ancora, regressivo». Un metodo non dissimile da quello che - a partire dal 
1887- Conan Doyle mette a disposizione di Holmes, coniugando in modo singolare lo stesso Kant, citato quasi alla lettera, con Cuvier: «la cosa essenziale - diceva - è riuscire a ragionare a ritroso" perché in tal modo un osservatore, "una volta afferrato un anello in una serie di incidenti, dovrebbe essere in grado di indicare con precisione tutti gli altri sia precedenti che successivi, proprio come Cuvier che descriveva un intero animale partendo dall'esame di un singolo osso.»

c) punta - più o meno esplicitamente- il proprio credito scientifico sull'idea di intelligibilità, di postdeterminazione: "L'intelligibilità — ha detto Von Wright- è un determinismo ex post facto.»

Primo quesito, allora: la scoperta di un nesso segreto tra A (dato) e B, proprio perché equivale alla estromissione di ogni apparente casualità, non porta a disintegrare le tesi rinvenibili nella lettera a Martha o a concludere che non solo la vita di Nathan Weiss, ma ogni vita - essendo dominata da una ferrea legge di determinazione- risulta "come composta da un poeta» e che "ogni catastrofe è una necessaria catastrofe»? La teoria psicoanalitica sembra dunque comportare o l'abrogazione di quella legge come legge territoriale della poetica o l'estensione di una legge della poetica all'intera esistenza.

Secondo quesito: Aristotele dice che A e B devono essere in un rapporto di verosimiglianza o di necessità. Una scienza, che adotti un metodo analitico, dovrebbe essere in grado di risalire fino a $\mathrm{B}$, fino alle condizioni sotto cui è possibile che A accada: in altri termini di individuare la causa necessaria (anche se non sufficiente) perché A si verifichi. Nel caso della psicoanalisi —e date le sue premesse - non esiste il rischio che, come in un'opera poetica, la verosimiglianza possa sostituirsi alla necessità? $\mathrm{O}$ peggio, come ha insinuato qualcuno, non può accadere che la teoria stessa si trasformi —al di fuori delle verifiche richieste dalle scienze classiche- in uno strumento per costruire verosimiglianze? per dire non : "poiché A è accaduto, è necessario che B sia accaduto"; $m a$ : "poiché $\mathrm{A}$ è accaduto, è verosimile (teoricamente verosimile) che $\mathrm{B}$ sia accaduto»? Dove può essere collocata allora una ragionevole linea di confine?

Terzo quesito : in cosa precisamente si differenzia il lavoro dell'analista dal lavoro del poeta? Se la non casualità è insita in ogni vita, sia l'uno che l'altro (lo psicoanalista e il poeta) mirano a metterla in luce. Ma già nel 1883 la specificità della letteratura è fortemente sottolineata: Freud la dichiara altra, irriducibile al suo resoconto.. E allora: si può dire che mentre la messa in intreccio,

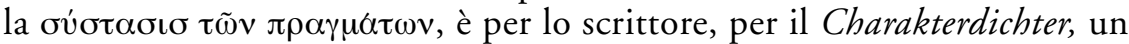
traguardo, qualcosa che egli si prefigge e che costruisce, usufruendo di una licenza tanto ampia da consentirgli di sostituire all'interno della sua creazione i legami di necessità con quella di verosimiglianza (o, se si preferisce, di fare della verosimiglianza una necessità fittizia), lo psicoanalista — dal canto suomira a quella Zusammensetzung come a un risultato della sua ricerca, un risultato reso obbligatorio dal determinismo assoluto del mondo reale, ma che non corrisponde in se stesso a nessuna intenzione esplicita, a nessun programma pregiudizialmente definito? È nella possibile conversione della necessità in 
verosimiglianza che dobbiamo dunque, o possiamo, riconoscere il privilegio (e anche il dono?) dello scrittore?

\title{
In cerca di una risposta spostiamoci nel 1895 e osserviamo Freud al momen-
} to di concludere uno dei casi clinici inseriti negli Studi sull'isteria

\begin{abstract}
Non sono stato sempre psicoterapeuta e ho fatto la mia esperienza medica con le diagnosi locali e con l'elettroprognosi, al modo stesso di altri neuropatologi, così che sento ancora io stesso un'impressione curiosa per il fatto che le storie cliniche che scrivo si leggono come novelle e che esse sono, per così dire, prive dell'impronta severa della scientificità. Devo consolarmi pensando che di questo risultato si deve evidentemente rendere responsabile più la natura dell'oggetto che non le mie preferenze; la diagnostica locale e le reazioni elettriche in realtà non valgono nello studio dell' isteria, mentre una rappresentazione approfondita dei processi psichici, come quella che si è abituati a ricevere dagli scrittori, mi permette, con l'impiego di poche formule psicologiche, di raggiungere una certa comprensione dell'andamento di un'isteria. Storie cliniche come queste vogliono essere giudicate come psichiatriche, ma presentano rispetto a queste ultime un vantaggio, e cioè l'intima relazione tra la storia delle sofferenze e i sintomi della malattia, relazione che nelle biografie di altre psicosi cerchiamo ancora invano.
\end{abstract}

Con queste parole si apre l'epicrisi del caso Elisabeth von R.: dal punto di vista strettamente retorico si tratta di una excusatio in piena regola. Freud mette avanti le proprie credenziali; ricorda il suo apprendistato con le diagnosi locali e l'elettroprognosi, in modo da potere presentare il suo attuale imbarazzo come una controprova. La correttezza dell'atteggiamento scientifico è dimostrata (non inficiata) dalla rinuncia all'impronta severa della scientificità, perché proprio quella rinuncia testimonia — su un piano più decisivo— che il ricercatore ha saputo adeguarsi all'oggetto, accantonando le proprie preferenze.

Da un lato troviamo storie psichiatriche, contrassegnate da quella «rigorosa impronta di scientificità», ma che non colgono la stretta relazione intercorrente tra i sintomi e l'evoluzione complessiva dei quadri clinici, storie che si limitano a un inventario e a una descrizione e che risultano assolutamente prive di "intreccio» : ci si limita, se vogliamo utilizzare due formule brillantemente escogitate da Forster nelle sue lezioni sul romanzo, a constatare che «prima morì il re e poi morì la regina», senza spingersi mai a dichiarare (o a congetturare) che "prima morì il re e poi, di dolore, morì la regina.». È proprio così, ad esempio, che sembrano essere costruite le «Osservazioni» di Krafft-Ebing. Dall'altro lato, le storie di Freud: storie cliniche che si leggono come novelle e mirano a mettere in luce non solo il sintomo, ma anche la sua genesi e le sue successive trasformazioni. La Einsicht si configura ora come il risultato di una rappresentazione analoga a quella a cui ricorrono gli scrittori.

Lasciate che insista perché si tratta di un punto decisivo. L'intelligenza del caso, infatti, viene fatta dipendere direttamente dalla forma narrativa, è una funzione di quella forma. Il racconto non è solo un espediente per presentare i fatti, e non è nemmeno una scelta tattica e congiunturale: è, viceversa, e a 
pieno titolo, un insostituibile strumento di conoscenza. Rappresentare gli eventi reali secondo i canoni della narrazione nasce, secondo Hayden White, dal desiderio di attribuire ad essi «la coerenza, l'integrità, la pienezza, l'organicità di una immagine della vita che è e può essere solo immaginaria. L'idea che sequenze di avvenimenti reali possiedano gli attributi formali delle storie che raccontiamo intorno ad avvenimenti immaginari non può avere la propria origine che nei desideri, nei sogni ad occhi aperti e nelle fantasticherie. E' forse possibile che il mondo si presenti alla nostra percezione nella forma di una storia ben costruita con un tema portante, un congruo inizio, un mezzo e una fine, e con una coerenza tale che ci permetta di scorgere in ogni inizio la fine che gli è propria?»

La risposta che Freud avrebbe dato a un simile interrogativo non è difficile da ipotizzare: chiedere al mondo di presentarsi nella forma di una «wellmade story» gli sarebbe certamente apparso illegittimo; e tuttavia gli sarebbe apparso non meno illegittimo immaginare un resoconto che parlasse di quel mondo e non avesse una forma latamente narrativa, la forma, magari, di una «badly-made story» non conforme a rigorosi principi di coerenza e ai protocolli aristotelici, ma - viceversa - all'ordine in cui si presentano molto spesso «desideri, sogni ad occhi aperti e fantasticherie». D'altronde, avrebbe aggiunto, la finzione è l'unico modo di rappresentare «una psicologia tridimensionale» proiettandola, al momento della scrittura, su una superficie piana. Non a caso la sua tecnica e la sua strategia di narrazione si modificano negli anni, riflettendo i successivi stati di una teoria e divenendo parte integrante di quella stessa teoria: quando la psicoanalisi non è ancora nata, al tempo degli Studi sull'isteria, l'attrezzatura di Freud è nello stesso tempo più tradizionale e più rudimentale tanto da indurlo a cautelarsi e ad attutire il potenziale discredito che avrebbe potuto derivargli da quelle che Krafft-Ebing definì una volta «favole scientifiche». "Favole scientifiche» immediatamente e potenzialmente scandalose: perché mandavano in frantumi abitudini consolidate, e perché sembravano erodere una rassicurante linea di confine su cui gli scienziati positivisti avevano giocato in parte la loro scommessa; e perché — scavalcando quel confine - preparavano la strada a una sorta di deflagrazione.

In realtà chi prende in mano la Psychopatia sexualis e legge dall'inizio alla fine "questo succedersi di ricerche viziose smisurate e, nella maggior parte dei casi, disperate», protese, aggiungeva Bataille, a conseguire «una soddisfazione in contrasto con tutto ciò che gli uomini possiedono di leggi, di convenzioni e di tranquillità", stenta sulle prime a rendersi conto del motivo per cui le ricerche di Freud hanno "turbato i sonni del mondo»: e non se ne renderà probabilmente conto fino a quando penserà agli oggetti «scandalosi» della psicoanalisi — alla sessualità infantile, al sadismo, al masochismo, alle pratiche onanistiche, alle perversioni etc. etc. Lo scandalo nasce piuttosto dalla forma narrativa adottata da Freud che, legando i sintomi alla storia del paziente- facendone un'emergenza e un'epifania —impediva di catalogarli e di neutralizzarli negli archivi medici: non erano, alla fine, "casi eccezionali», erano - a pieno titolo- storia di tutti. Non era più possibile guardare quei casi come mostruo- 
sità ed escrescenze immunizzate da uno statuto di eccezione e di alterità; era «il quotidiano vivere associato», erano le leggi e i sonni di tutti a essere minati alle origini. Il mostro perdeva di colpo la sua identità e la sua funzione di capro espiatorio: si aggirava tra gli uomini, era in "padri, fratelli e figli, mogli e madri, nel sangue della stessa stirpe» e nel patrimonio di ogni singolo individuo.

La tecnica narrativa di Freud è ben più libera e più consapevole quando, nel 1901, pubblica Frammento di un'analisi d'isteria (1901), che è il primo dei grandi casi clinici scritto alla luce della teoria psicoanalitica. La narrazione è sottoposta a radicali modificazioni. La mutata prospettiva teorica porta a scelte formali inedite, a una messa in scena impensabile prima della Interpretazione dei sogni che ha visto la luce due anni prima.

Freud procede con molta cautela; è consapevole della novità e si affretta a dichiarare l'imperfezione dei risultati: «non ho ancora risolto — scrive nella Premessa - il problema di come fissare, per esporla in seguito, la storia di un trattamento di lunga durata»: E, all'inizio del Poscritto, ribadisce che si tratta di un frammento (per giunta «ben più incompleto di quanto il titolo lasciasse presagire»), non di una storia compiuta, e che le numerose lacune non sono casuali. Sono, viceversa, l'inevitabile conseguenza di un'attrezzatura tecnico-narrativa che non riesce (non riesce ancora) ad adeguarsi alle richieste di una teoria rivoluzionaria, a cui non possono più bastare gli strumenti di stampo essenzialmente naturalistico di cui Freud si serve negli Studi sull'isteria.

Nello stesso poscritto Freud discute per la prima volta in modo approfondito il significato e il valore della traslazione (Übertragung): "è una riedizione, - dice- una copia degli impulsi e delle fantasie che devono essere risvegliati e resi coscienti durante il progresso dell'analisi.» Secondo due modalità: quella della ristampa, che riproduce fedelmente i modelli, e quella del rifacimento, realizzato «artisticamente» tramite il ricorso a procedimenti come la Milderung (mitigazione) e la Sublimierung (sublimazione) che a più riprese Freud indicherà come tipici dell'elaborazione letteraria.

Anche nel passaggio dal reale svolgimento delle sedute al resoconto analitico, che è una sorta di traslazione, si profilano due possibilità: il «modello» può essere ristampato (un esempio verrà fornito dal Diario dell'uomo dei topi), o sottoposto a un radicale rifacimento, come accade nei Casi clinici. Allora il narratore, che non coincide affatto con il terapeuta, ma è solo un suo omonimo, si concede ampie libertà: libertà di taglio, di «intrusione» (nel senso definito da Georges Blin), ma soprattutto di «messa in intreccio», di affabulazione e di rettifica dell'ordine cronologico.

Il resoconto — scrive Freud nella Premessa al Frammento — non è di una fedeltà assoluta, fonografica, ma gli si può riconoscere un alto grado di attendibilità. Nulla di sostanziale è stato mutato, se non in alcuni punti il succedersi delle spiegazioni, che ho così disposto per amore della coesione (Zusammenhang).

L'esito più spettacolare della regia di Freud è rappresentato, in questa cir- 
costanza, dalla messa in scena, alla fine, di due grandi sogni che, registrati "letteralmente subito dopo la conclusione delle sedute», nel tempo reale dell'analisi erano "riferiti uno alla metà, l'altro alla fine della cura.» La dislocazione consente a Freud di costruire il proprio resoconto in base a un protocollo inedito, che riserva alla produzione onirica il valore di un luogo di epifania.

Tra la Premessa e il Poscritto trovano posto tre atti: Lo stato clinico, Il primo sogno, Il secondo sogno. Quasi alla fine del primo atto, Freud sembra avere raggiunto una spiegazione soddisfacente: l'amore di Dora per il padre si è trasferito su K., una figura paterna. I conti, in tal modo, tornano perfettamente: tutto si tiene e il caso sembra avere raggiunto una sua conclusione esemplare ed esteticamente ineccepibile. Ma, a questo punto, Freud è costretto ad annunciare un rovesciamento inatteso e radicale, e lo fa con parole di cui è impossibile sopravvalutare l'importanza:

Devo ora parlare di un'altra complicazione, a cui certo non dedicherei spazio alcuno se fossi un'artista che deve inventare un simile stato d'animo in un racconto, invece di un medico che ne deve fare la dissezione. L'elemento a cui ora alluderemo non può che offuscare e dissolvere la bellezza, la giusta misura poetica del conflitto che ci è lecito ascrivere a Dora; esso verrebbe a buon diritto sacrificato dalla censura dell'artista che, del resto, quando appare nelle vesti di psicologo, semplifica e astrae. Ma nella realtà che io mi sforzo qui di descrivere la complicazione dei motivi, il cumulo e la combinazione degli stati d'animo, in una parola la sovradeterminazione, costituiscono la norma.

Freud si trova a un punto decisivo ed è costretto a varcare una linea di confine. La psicologia degli scrittori — dice— «semplifica e astrae»: può accontentarsi di ricostruzioni parziali e, in ogni caso, non è tenuta in linea di principio a rendere nessun conto alla realtà, perché deve limitarsi al rispetto di regole formali di costruzione. Il contrasto con le dichiarazioni che avevano trovato nel caso Elisabeth von R. è molto netto, anche se allora Freud si era limitato a sottolineare la tecnica della messa in intreccio e non si era esplicitamente pronunciato sulla effettiva profondità psicologica raggiunta dagli scrittori di racconti. La costruzione fin qui elaborata, e che sembra finalmente avere raggiunto il proprio punto di equilibrio, deve essere distrutta: la legalità aristotelica («bisogna preferire i fatti impossibili ma verosimili a quelli possibili ma incredibili») è, a partire da questo momento, drasticamente sospesa. Freud deve mettere insieme (ouviotaval, zusammensetzen) il suo caso, il suo resoconto par-

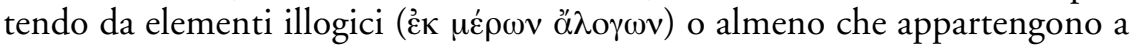
una altra logica. Non è solo la spiegazione psicologica tradizionale che crolla; è anche la forma narrativa che si rompe e deve essere rinnovata. Freud gioca allora - sui due piani - la carta del sogno.

Più tardi, nel Caso clinico dell'uomo dei lupi (1918), Freud, dopo avere ripetutamente sottolineato la difficoltà di intrecciare insieme storia della malattia e storia del trattamento, osserva che il lavoro del resoconto analitico trova «una barriera naturale quando si tratta di portare sul piano descrittivo bidimensio- 
nale una struttura in sé pluridimensionale.» Proprio a un tentativo di scavalcare o di aggirare quella barriera naturale sembra corrispondere, nel caso Dora, la collocazione strategica dei due grandi sogni, dove il tempo narrativo lineare esplode e si dispone in corone irregolari intorno a due nuclei forti. "Se fossi uno scrittore mi fermerei qui»: non essendo uno scrittore, ma uno scienziato, Freud si trova nella necessità di organizzare una inedita strategia narrativa, di inventarsi — alla lettera - una «scrittura». Non sono più, a guidare, i suoi passi

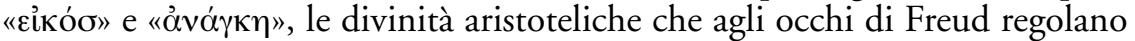
il lavoro del Charakterdichter. La frattura è —o dovrebbe essere- decisiva, perché a Freud poco importa se — quasi negli stessi anni- qualcuno comincia a screditare quelle divinità e a parlare di «stupidissima verosimiglianza»; e poco importa se già scricchiolano modelli narrativi che, nel giro di poco tempo, andranno in frantumi. Ai suoi occhi il modello della narrazione letteraria resta saldamente, ideologicamente ancorato a quello della grande «tradizione» che ha imparato a conoscere fin dai tempi dell'Accademia Spagnola.

Negli ultimi anni della sua vita Freud non esiterà a riconoscere che Gradiva di Wilhelm Jensen è un racconto di qualità non eccelsa, assimilabile a quei prodotti medi che costituiscono una variante scritta e opportunamente manipolata del sogno ad occhi aperti, e che avevano attirato la sua attenzione nel saggio Il poeta e la fantasia. Si tratta, forse, di un giudizio a posteriori: né si può escludere che, a determinarlo almeno in parte, abbiano contribuito le reticenze di Jensen di fronte alle domande con cui Freud, complice Carl Gustav Jung, aveva cercato, dopo la sua «rilettura», di stringerlo d'assedio.

In ogni caso, quando Freud si misura col testo di Jensen, nel 1906, e lo sottopone a un vero e proprio lavoro di riscrittura, passando alle spalle dei personaggi e trattandoli - loro e il loro sintomi- come se fossero personaggi in carne ed ossa, e come se quei sintomi fossero reali, nel suo apprezzamento non c'è ombra di riserve: né sulla plausibilità psicologica di Norbert Hanold; né sull'efficacia della talking cure, intrapresa da Zoe Bertgang tra le rovine di Pompei; né sulla costruzione dell'intreccio.

Che il racconto sia fragile e di scarsa tenuta lo potrebbe forse dimostrare la facilità con cui l'interprete riesce ad appropriarsene, con cui installa, al posto di un autore esautorato e defraudato, il suo laboratorio da campo. Jensen, comunque, o il suo fantasma, appare un prezioso alleato. Gli vengono attribuite saggezza, veggenza e un'infallibile insight, che si manifestano tra l'altro in una sorprendente descrizione della genesi del delirio. Ma una simile descrizione, si chiede Freud, può «reggere di fronte al giudizio della scienza?»

Dobbiamo dare una risposta che forse è inattesa [...] La scienza non regge di fronte all'opera del poeta. Fra le premesse d'ordine ereditario-costituzionale e le produzioni del delirio che sembrano emergere ben finite, la scienza lascia sussistere un vuoto, che troviamo invece riempito dal poeta. Essa, la scienza ufficiale, non ha ancora intuito il significato della rimozione, non riconosce ancora che volendo spiegare il mondo dei fenomeni psicopatologici è assolutamente indispensabile ricorrere all'inconscio, non cerca la radice del delirio 
in un conflitto psichico e non ne afferra i sintomi come formazioni di compromesso. Dobbiamo allora dire che il poeta si trova contro la scienza intera? No, questo no....almeno se è lecito all'autore considerare anche i propri lavori come qualche cosa che appartiene alla scienza.

Freud sembra riprendere il discorso là dove l'aveva interrotto aprendo l'epicrisi del caso Elisabeth von R. La scienza, di cui Freud parla in questa circostanza, non è la scienza nel suo complesso: è la psichiatria, che si limita a pure e semplici descrizioni dei quadri clinici e che -ignorando la rimozione e non riconoscendo l'inconscio- da un lato non interpreta i sintomi come funzioni di compromesso, dall'altro non può ricostruirne la storia e non può quindi che restare (quasi programmaticamente) al di qua di ogni possibile spiegazione. Agli occhi di Freud gli psichiatri si comportano come chi, di fronte a fenomeni inesplicabili, è disposto a prenderne atto, ma non a cercare di capirne le ragioni e le modalità. Nei loro confronti l'atteggiamento di Freud è apertamente provocatorio, quasi sarcastico: con una decisione spavalda colloca se stesso, e la sua scienza, dalla parte della letteratura, limitandosi a sottolineare che esiste una differenza sul piano delle formulazioni tecniche: ma Jensen "ha visto tutto e ha raccontato tutto».

Ha raccontato. Ha spiegato. Ha intuito la rimozione. Ha lasciato all'inconscio la sua parte. Freud non può fare diversamente: ogni altra scelta —ogni abbandono del piano narrativo a favore del piano descrittivo- risulterebbe fallimentare tanto per la teoria, quanto (e peggio ancora) per la terapia. Il racconto, in quanto strumento di conoscenza, può essere anche mezzo di guarigione.

Ventotto anni dopo, nel 1934, Freud scrive L'uomo Mosé, «un romanzo storico» - come lo definisce lui stesso in una nota e in una lettera del 30 settembre a Arnold Zweig. Per capire il valore di quelle parole bisogna però rifarsi a un' altra lettera allo stesso corrispondente, scritta qualche mese prima (il 12 maggio), in cui troviamo una confutazione molto determinata del progetto di scrivere una biografia di Nietzsche che Zweig gli aveva esposto il 28 aprile.

Freud parte dal problema della libertà degli scrittori creativi di fronte alla realtà storica: "La mia posizione — dichiara preliminarmente- è molto conservatrice.»

Là dove, nella storia o nella biografia sia presente una lacuna irrimediabile, il poeta può spingersi avanti e cercare di indovinare come sono andate le cose. In un paese inabitato egli può installare le creature della sua immaginazione. Anche quando la storia è nota, ma troppo lontana ed estranea alla coscienza comune, può metterla da parte. Non si può dunque obiettare a Shakespeare che intorno all'anno mille Macbeth era un re di Scozia giusto e popolare.

Le regole, continua Freud, mutano radicalmente quando ci si trova davanti a un personaggio contemporaneo o quasi contemporaneo come Nietzsche: in tal caso lo scrittore è tenuto a fornire un ritratto scrupolosamente somi- 
gliante e, siccome non può far posare di fronte a sé il soggetto, deve affidarsi a una scrupolosa raccolta di materiali, su cui dovrà esercitare le proprie capacità di approfondimento e di comprensione. E conclude:

Provi un po' a pensare cosa ce ne faremmo di un Friedrich Nietzsche immaginario?

Le leggi, a cui Freud ritiene di doversi attenere nella redazione del suo romanzo storico, risultano chiaramente da questi principi e trovano una conferma nel testo: lo articolano, stabiliscono una ininterrotta catena di punti di riferimento. La figura di Mosé, ci viene detto, è avvolta nelle nebbie di una tradizione deformante: la sua leggenda è circondata dagli indizi inconfondibili di «un'opera secolare e inesausta», di una rielaborazione tendenziosa, stratificata, tale da sfidare «vittoriosamente ogni tentativo di riportare alla luce il nucleo della verità storica che ad esse è sotteso.» I materiali sono incompleti, lacunosi, molto spesso inaffidabili; e se è vero che nella "deformazione di un testo", come nel compimento di un delitto, la difficoltà consiste nel non lasciare tracce dietro di sé, questo non toglie che quelle tracce costituiscano delle spie, degli indizi significativi che fanno scricchiolare l'edificio della tradizione, ma che non conducono automaticamente, e con assoluta sicurezza, all'accertamento dei fatti come sono accaduti.

Si possono certo sovrapporre i diversi frammenti e le diverse versioni, come ha fatto Rank seguendo il metodo di Galton, per riportare alla luce i lineamenti fondamentali della «leggenda dell'eroe»; si possono disporre i pezzi sul tavolo e cercare di incastrarli gli uni negli altri come in un gioco di pazienza, ricostruendo congetturalmente, se è il caso, i pezzi mancanti e necessari perché il puzzle alla fine risulti compiuto. Né mancheranno i supporti analogici per passare da un campo all'altro e per istituire connessioni tra la psicologia individuale e la psicologia collettiva. Questo non toglie che la soluzione non potrà che basarsi sulla «legge della minima resistenza, preferendo quelle ipotesi a cui si potrà attribuire il più alto grado di verosimiglianza.» Ma, avverte Freud all'inizio del «secondo saggio»,

la verosimiglianza, per quanto seducente, non ci garantisce dall'errore; anche quando tutti gli elementi di un problema sembrano adattarsi l'uno all'altro, come i pezzi di un incastro, occorre riflettere che il verosimile non necessariamente è il vero e che la verità non sempre è verosimile.

Cautela aristotelica e cautela indubbiamente apprezzabile in chi dichiara di non volersi confondere con "gli scolastici e i talmudisti», sempre pronti ad appagarsi della "loro sottigliezza», ma non sufficiente certo a impedire che Freud — con il suo romanzo storico — venga assimilato a coloro a cui Aristotele si rivolgeva: agli scrittori che devono puntare alla coerenza dei loro edifici e che in quella coerenza - e non altrove - troveranno la soluzione, la $\lambda v \sigma \iota \sigma$, di cui vanno in cerca. Così che alla fine lo spirito critico di Freud dovrà guardare al suo lavoro come "a una ballerina in equilibrio sulla punta di un piede.» 
Equilibrio reso ancor più precario dalla natura ibrida di quel lavoro, che Freud sottolinea in una breve nota ritrovata, per l'edizione italiana, da Pier Cesare Bori:

Come l'unione sessuale di cavallo e asino dà origine a due diversi ibridi, il mulo e il bardotto, così la fusione tra scrivere storia e libera invenzione fa sorgere diversi prodotti, che, sotto la designazione comune di «romanzo storico», vorranno esser valutati ora come storie, ora come romanzi.

Freud non può sfuggire alla propria consapevolezza ed è allora che se ne viene fuori con una presentazione di credenziali sorprendentemente simile a quella che, in anni lontani, aveva avanzato al momento di aprire l'epicrisi del caso Elisabeth von R., e che sembra rappresentare una drastica inversione di marcia rispetto alle posizioni acquisite fin dal 1906. Dopo avere ricordato che mentre alcuni narratori accentuano la parte dell'invenzione e altri quella della verità storica, che altri ancora sembrano capaci di contemperare le due esigenze e che la creazione poetica si insinua inevitabilmente anche nel lavoro degli storici, pur contro le loro dichiarate intenzioni, Freud prosegue:

Se tuttavia io, che non sono né ricercatore storico né artista, definisco uno dei miei lavori come «romanzo storico», ciò significa che questo termine consente ancora un altro impiego. Sono stato educato all'osservazione accurata di un determinato ambito di fenomeni e facilmente per me all'elaborazione letteraria e all'invenzione si collega la macchia dell'errore.

La mia intenzione più prossima era di acquisire una conoscenza della persona di Mosé, il mio fine ultimo di contribuire alla soluzione di un problema ancor oggi attuale...

Qui, tuttavia, non è il «fine» più o meno ravvicinato di Freud che importa; importa invece la necessità, in cui ancora una volta viene a trovarsi, di riconoscere l'ineluttabilità circostanziale della forma narrativa: è stato costretto ad accettarla, a piegarsi ad essa nonostante i pregiudizi, nonostante il giuramento - formulato in anni ormai molto lontani- di abbandonare la letteratura.

Allora — in quei tempi, quelli dell'Accademia spagnola - Freud aveva inviato a Silberstein «un racconto biblico in cui aveva manipolato artisticamente il vissuto». Quel racconto si era smarrito insieme con la lettera: all'amico, che gli chiedeva di riscriverlo, Freud aveva risposto con ironica enfasi:

Non parlarmi di surrogati. Se un giorno il sole andasse in frantumi e vivessimo al buio, che surrogato potresti trovargli? Se l'oceano si prosciugasse e le sorgenti del cielo si inaridissero, che surrogato dell'acqua suggeriresti?

Il saggio su Mosé può apparirci come l'impossibile surrogato di un testo perduto, una sorta di elegia ebraica scritta, quando «il sole sta per andare in frantumi», da un vecchio che ha riesumato ancora una volta — per l'occasionela bacchetta di Prospero. 